\title{
Vorwort zur achten Auflage.
}

Zur Einführung lieser neuen Auflage, welche sorgfiltig auf Grund ler neuen Literatur durchgesehen wurde, gestatte ich mir, aus den früheren Vorreden einige Erläuterungen zu wiederholen.

Der vorliegende Grundrib ist nicht bestimmt, ein Lehrbuch der Botanik zu ersetzen, noch viel weniger, Vorlesungen über diesen Gegenstand ïberflüssig zu machen; er soll denjenigen, welche die Botanik nicht als Fachstudium betreiben, als Leitfaden dienen und als Hilfsbuch neben den Vorlesungen benützt werden, um das Gerippe dieser Wissenschaft zur Hand zin haben.

Auf unseren Hochschulen wird eine Reihe von Lehrbüchern der Botanik benutzt, welche das Ziel des wissenschaftlich-botanischen Unterrichts in etwas verschiedener Form verfolgen; die Lehrbücher von Stras. burger, Giesenhagen, Prantl-Pax. Jedes hat seine Eigentümlichkeit. Die allgemeinste Verbreitung hat sich das von Strasburger und seinen Mitarbeitern verfalite Buch errungen. Vollstindigkeit in der Beschrinkung, wissenschaftliche Höh bei didaktischen Streben, bilden seine Vorzüge. Es hiitte somit wenig Wert, diesen genannten noch ein weiteres Lehrbuch zuzufügen. Fin literarisches Bedürfnis liegt dagegen anderswo. DaB die Studierenden einen kürzeren Auszug aus dem Gesamtwissen nötig haben, beweisen die Kollegienhefte, welche bekanntlich auch für die Prüfungen wieder hervorgeholt zu werden pflegen. Aber diese Aufzeichnungen sind meist lückenhaft, unübersichtlich und auch vielfach unrichtig. Das vorliegende Hilfsbuch hat von Anfang an das Ziel verfolgt, im AnschluB an gehörte Vorlesungen dem Studierenden eine kurze und richtige Ubersicht von dem, was er unbedingt im Kopfe haben mul, um tiefer in die Wissenschaft eindringen zu können, zu bieten. $\mathrm{Da} B$ es auch nach dem Studium dem Lehrer als sehr brauchbarer Leitfaden dienen kann, wurde mir vielfach versichert. Dementsprechend ist alle Mühe auch bei der neuen Auflage darauf verwendet worden, das Buch dem Fortschritt der Wissenschaft gemäß zu ergïnzen, damit es mit den größeren Lehrbüchern, bei äußerer Bescheidenheit, an innerem Wert Schritt hiilt.

Die Abschnitte der allgemeinen Botanik schlieBen sich an die auf Seite 2 genannten grundlegenden Handbücher an. Über den systematischen Teil ist etwas mehr zu sagen. Das System, besonders das der Blütenpflanzen, bildet noch, wie ehedem, wegen seiner in der Natur. 
der Sache liegenden Bewegung und Umgestaltung, eine Schwierigkeit für den Anfünger. Gegenwärtig hat man sich allgemein dem Engler. schen System, wie es in dessen "Syllabus der Pflanzenfamilien" 6. Auflage 1909 aufgestellt ist, angeschlossen.

Trotzdem habe ich mich nicht entschließen können, dies wissenschaftlich strenge System für die Anordnung in diesen Buche zu bcnutzen. Dem Anfänger, der sich zunïchst im Trennen übt, erscheint die enge Zusammenfassung mancher Familien, wie 2. B. in den Englerschen Reihen der Rosales und Tubifloren erschwerend, ebenso, wie die Auflösung anderer Reihen in zahlreiche kleine Abteilungeu. Pädagogisch crscheint mir daher richtiger, zuerst mit einem einfacheren System zu beginnen. Um nicht der Willkür zu verfallen, ist es natürlich und wissenschaftlich zugleich, um zum Einfacheren zu gelangen, der Geschichte zu folgen und zum Eichlerschen System zurückzugreifen. Ich durfte das um so mehr tun, als der deutsche Meister der Systematik A. Engler in dem Vorwort zu seinem Syllabus ebenfalls das Eichlersche System als das für den Anfänger leichter faßliche bezeichnet. Über sein eigenes System fügt er hinzu: „Es weicht mehrfach vou dem des Eichlerschen Syllabus ab, es liegt aber auch ihm, sowie den Systemen von A. Braun und Eichler das System von A. Brongniart zugrunde." Um den Anfünger einen Vergleich beider Systeme zu ermöglichen und seinen Blick zu erweitern, habe ich das Englersche System der Phanerogamenfamilien in Tabellenform aufgenommen.

Ganz unverïndert konnte ich freilich auch Eichlers System nicht lassen, so wurde die Abteilung der Hysterophyten aufgegelen und bei den Kryptogamen mußten, dem wissenschaftlichen Fortschritt gemäb, ziemlich weitgehende $\ddot{\Lambda}$ nderungen getroffen werden.

Wenn auch heute jeder Darstellung des Systems das phylogenetische Prinzip zugrunde liegen soll, so stört seine allzustrenge Durchführung nicht selten die praktische Übersichtlichkeit, welche durch die vergleichende Morphologie oft leichter erreicht wird. Und da auch die Führer der systematischen Wissenschaft beide Prinzipien verbinden, so ist dies in einem Hilfsbuche um so mehr geboten, wo es das Bedürfnis nach Klarheit erfordert.

Gießen, März 1910.

Dr. A. Hansen. 